\title{
Dynamiques d'engagement des enseignants débutants en formation et appréhension des processus identitaires
}

\section{Guillaume Serres, Nicolas Perrin, Serge Leblanc et Luc Ria}

En référence aux enjeux de ce numéro thématique, cette contribution propose de rendre compte d'expériences de formation d'enseignants débutants au cours desquelles sont repérables des transformations de leur engagement. L'approche privilégiée accorde une importance particulière à la dimension dynamique des phénomènes dès lors décrits sur la durée à partir d'études longitudinales. Trois modalités de transformation ou reconfiguration de l'engagement des enseignants débutants ont été caractérisées sous la forme de "sorties d'impasses», de "transitions» et de «reconstructions massives». Une meilleure compréhension des processus identitaires en jeu lors de ces reconfigurations nous semble a même d'ouvrir des pistes prometteuses pour la formation.

\section{Introduction}

La problématique identitaire fait largement question dans la réflexion sur la formation des enseignants. Considérée comme évidente et nécessaire, elle reste à ce jour tout autant problématique. Malet (2008) souligne que son invocation tous azimuts et sa connotation statique ne sont pas étrangères au flou qui l'entoure. Dans les faits, cette problématique est souvent associée au caractère abrupt de la prise de fonction et de responsabilité (Baillauquès, 2002).

En France, les réformes successives de la formation des enseignants insistent toutes sur la nécessité d'une entrée progressive dans le métier. Les états généraux pour la formation des enseignants qui se sont tenus au cours des dernières réformes dites de la mastérisation, ont également souligné l'importance d'envisager la question de l'identité professionnelle dans la durée. Des propositions ont été faites pour offrir des stages d'observation dès les premières années de licence aux étudiants qui envisagent de devenir des enseignants. De plus, la posture d'observateur a été jugée insuffisante pour se projeter «pilote» d'une classe, en ce sens un stage de pratique accompagnée le plus tôt possible (premier semestre de master) a été revendiqué. Les textes-cadres pour la formation insistent donc sur l'importance d'une professionnalisation progressive et sur l'encadrement des 
formés dans les différents lieux de formation.

Au-delà de cette recherche d'une immersion progressive dans le milieu professionnel, plusieurs façons d'appréhender et de travailler les questions identitaires nous semblent repérables dans les dispositifs de formation des enseignants en France. La question de l'identité prend parfois la forme d'une réflexion sur la personnalisation de la formation (Baillauquès, 2002). Pour Gouillaud (2001), le risque majeur est de renforcer des savoirs et des pratiques développées par les enseignants débutants à partir de leur expérience d'élève au détriment de la construction de savoirs nouveaux indispensables pour l'exercice du métier. L'identité peut être encore envisagée comme mise en mouvement de l'apprenant vers un nouveau rapport au savoir (Barbier, Bourgeois, De Villiers \& Kaddouri, 2006). Selon cette approche de l'identité, le volet des acquisitions cognitives est premier et supplante toutes les dimensions de l'activité, laissées dans l'obscurité. Plus largement, on peut constater que l'identité est plus souvent envisagée sous l'angle des acquisitions (processus d'acquisition de savoirs, de compétences, de gestes professionnels, etc.) sans qu'elle soit considérée conjointement sous l'angle de la participation (processus d'intégration d'une communauté professionnelle porteuses de règles et de valeurs). A nos yeux ces deux dimensions sont indissociables et leur prise en compte conjointe en formation nous semble incontournable.

Dans ce contexte de réforme, la formation doit composer avec un temps de plus en plus limité, mais aussi avec les difficultés liées à la mise en place et à l'encadrement des stages. On peut constater la mise en œuvre de dispositifs spécifiquement pensés pour composer avec les nouvelles données de la formation. Des dispositifs de formation se sont par exemple appuyés sur l'outil vidéo en vue de se rapprocher de la réalité des classes (Ria, Serres \& Leblanc, 2010). D’autres expériences ont été menées pour faire intervenir des acteurs professionnels afin de simuler des interactions parents-professeur. D'importants efforts sont également produits pour travailler la cohérence entre terrain professionnel et institut universitaire de formation notamment par la mise en place de collaborations plus étroites entre les formateurs des établissements scolaires qui accueillent des enseignants débutants en stage et les superviseurs universitaires chargés des enseignements les plus professionnalisant. Ceci est observable en Suisse (Périsset, 2006), au Québec (Boutet \& Pharand, 2008) ou en France (Chaliès, Bertone, Flavier \& Durand, 2008).

\section{Problématique}

Cette contribution se focalise sur le repérage de moments de formations porteurs d'enjeux identitaires et sur la caractérisation de l'activité des formés au cours de ces derniers. Comme le soulignent Rayou et Ria (2009), quelques enjambées pour faire le tour du bureau, depuis le banc de l'élève jusqu'à l'estrade profes- 
sorale ne suffisent pas pour devenir enseignant. Ce déplacement physique de la position d'élève à celle de l'enseignant s'accompagne de multiples transformations identitaires dont l'étude ouvre des pistes de discussion estimées cruciales pour l'accompagnement des enseignants dans leurs débuts dans le métier. C'est dans cette problématique que nous nous inscrivons en focalisant notre étude sur certaines expériences qui ont reconfiguré les façons d'être, d'agir, de percevoir, d'éprouver et de connaître des enseignants débutants avec lesquels nous avons travaillé. Nous proposons ainsi d'analyser les processus par lesquels un acteur lie entre elles des expériences diverses en une trajectoire cohérente afin de lui donner une certaine unité (Durand, 2008 ; Serres, 2006).

Plus largement, cette contribution s'inscrit dans la lignée des travaux de recherche qui analysent l'activité des enseignants dans son devenir (Malet, 1998, 2008), à des étapes de leur parcours de formation comme leur entrée dans le métier (Goigoux, Ria \& Toczeck, 2010; Ria, 2006, 2011; Rouve \& Ria, 2008; Saujat, 2004).

Le but de notre contribution est d'identifier dans les trajectoires des débutants des passages qui témoignent à nos yeux des difficultés de prise en compte des enjeux identitaires dans les dispositifs de formation. Par la suite nous proposons en discussion de souligner des pistes d'action pour la formation qui consisterait à: a) jouer sur la réversibilité des situations, b) accélérer la survenue de processus jugés favorables, c) penser la progressivité des questions professionnelles, e) penser la complexité des dynamiques simultanément à l'œuvre.

\section{Cadre théorique et méthodologique}

La recherche a été menée selon le cadre théorique et méthodologique du cours d'action (Theureau, 2004, 2006, 2009). L'objet d'analyse privilégié est le couplage entre l'acteur et son environnement que nous spécifions par la notion d'engagement (Theureau, 2004). L'engagement de l'acteur dans la situation traduit l'ouverture/clôture des possibles pour l'acteur. Documenté à un instant «t», il permet de décrire un ensemble de qualités, propensions, dispositions qualifiant, à chaque instant, la relation ou couplage qu'un acteur entretient avec son environnement. Si l'objet d'analyse est bien le couplage de l'acteur avec son environnement, l'étude a consisté à rendre compte de l'histoire de ces couplages au cours d'expériences repérées comme porteuses d'enjeux identitaires particuliers.

Les données sont issues de plusieurs recueils qui avaient pour objectif de documenter l'expérience des enseignants débutants du premier et du second degré de l'enseignement scolaire sur des empans temporels longs en France. Elles ont été recueillies au cours de leur ultime année de formation ou au cours de la première année d'exercice professionnel en temps que titulaire. Au total, le recueil concerne 15 professeurs de différents niveaux scolaires mais aussi de différentes disciplines scolaires pour ceux appartenant au second degré. 
L'investigation a été réalisée à partir d'observations et d'entretiens d'autoconfrontation. De façon systématique, ces observations et ces entretiens ont été utilisés comme supports complémentaires de description.

Dans le cadre de cet article, nous nous sommes attachés à une description plus détaillée d'expériences qui ont joué, du point de vue des débutants, un rôle clef dans le décours de leur formation. L'analyse a porté spécifiquement sur l'analyse des propriétés de ces courtes expériences marquées par des transformations de leur façon d'être, d'agir, de percevoir, d'éprouver et de connaître, de manière à caractériser par la suite les reconfigurations de leur engagement. Les processus identitaires dans cette perspective sont envisagés à partir d'une description de bascules dans le couplage qui lie l'acteur à son environnement. Ils sont appréhendés selon notre cadre théorique et méthodologique par l'analyse de ce que Theureau (2006) désigne par la dynamique des ouverts. L'activité est envisagée comme un composite de permanence et de changement (Durand, 2009), il s'agit de rendre compte des recompositions de ces permanences et changements en s'attachant à la façon dont les acteurs typicalisent leur activité et tentent de donner de l'unité aux expériences qu'ils vivent.

Nous avons identifié au total pour nos 15 professeurs 28 extraits que nous avons regroupés en trois ensembles: 5 extraits ont été catégorisés sous l'intitulé «sorties d'impasses», 15 extraits sous l'intitulé «transitions de phases» et 8 extraits sous l'intitulé «reconstructions d'édifices». Cette quantification donne une idée du recueil de données mais ne permet pas d'établir de tendance sur la survenue des phénomènes décrits.

Ces reconfigurations de leur engagement présentent une ou plusieurs des caractéristiques suivantes:

a) elles amènent les acteurs à reconsidérer des pratiques de classe stabilisées depuis plusieurs semaines, voire plusieurs mois;

b) elles amènent une reconfiguration (une remise en jeu) de leurs façons d'être, agir, percevoir, éprouver et connaître jusqu'alors envisagées comme stabilisées et durables;

c) les reconfigurations marquent un contraste fort entre les façons d'être des enseignants en amont et en aval de ces expériences;

d) les reconfigurations envisagées renouvellent (réorientent) les possibilités de développement de l'activité.

\section{Résultats}

Trois extraits d'analyse sont ici synthétisés. Les extraits choisis sont de bons représentants pour décrire les trois modalités de transformations de l'activité distinguées.

Un premier ensemble d'extraits analysés a été caractérisé sous la modalité «sorties d'impasse» dans la mesure où l'engagement des débutants avant la 
survenue de ces expériences semble stable et contraint avant de bifurquer de façon majeure et de s'ouvrir sur de nouvelles possibilités de développement.

Un autre ensemble d'extraits a été caractérisé en référence à la modalité «transitions de phases» dans la mesure où les transformations observées semblent s'inscrire dans une certaine continuité avec l'activité passée de l'acteur tout en ouvrant de nouvelles possibilités de développement.

Un troisième ensemble d'extraits «reconstructions d'édifices» a été caractérisé pour les modalités de transformations de l'activité supposant pour les acteurs la reconsidération de pratiques de classes stabilisées souvent sur un temps conséquent (plusieurs semaines à plusieurs mois), pratiques devenues constitutives de leur façon de faire habituelle.

\section{Sorties d'impasses: Léa, professeure stagiaire de Lettres-Histoire}

L'extrait suivant concerne Léa, professeure stagiaire de Lettres-Histoire en lycée professionnel. Le temps de formation en centre (IUFM) évoqué s'est déroulé cinq mois après la rentrée des classes. En s'appuyant sur la plateforme de formation Néopass@ction développée au sein de l'Institut Français d'Éducation, un formateur développe une analyse de l'évolution des gestes professionnels des débutants lors des premières semaines de prise de fonction. Un épisode est décrit par Léa de façon particulière dans le décours de cette séance de formation en centre: "Là, je me dis qu'en fait on peut rater sa première heure de cours sans que l'année soit fichue pour autant, [Léa, Lettres-Histoire, début décembre]. A cet instant, Léa semble ressaisir un ensemble d'éléments de son expérience pour les considérer à nouveaux frais. Le plus ancien est relatif à sa première heure de cours en tant que professeure stagiaire ayant la responsabilité d'une classe. Léa estime avoir raté ce moment face aux élèves. Depuis elle considère qu'il n'est pas possible d'infléchir cette situation. La comparaison en formation de deux extraits vidéo d'un même professeur débutant contrôlant le groupe classe de façon très contrastée en tout début d'année l'amène à revoir sa façon de considérer son activité à venir. Amenée par le chercheur à commenter plus dans le détail la réflexion qui s'opère chez elle lors de ce temps de formation, elle précise: "Jusqu'ici, j'avais vraiment l'impression que ce qu'il me restait à faire cette année, c'était de limiter les dégâts et d'attendre la prochaine rentrée pour essayer autre chose. Là, je me dis qu'il est possible d'inverser la vapeum. Considérant vraie l'affirmation selon laquelle «rater sa première heure de cours c'est rater son année», l'enseignante débutante est restée pendant plusieurs semaines dans cette situation qu'elle jugeait irréversible. La confrontation à une description d'évolutions possibles de l'activité de débutants et à l'analyse de ces évolutions par le formateur semble l'amener à reconsidérer cette «doxa» admise de façon quasi unanime par les débutants. L'analyse d'un point de vue en extériorité nous amène à considérer que ce moment de formation participe à une reconfiguration majeure de l'engagement de cette stagiaire. Ce dernier semblait fortement conditionné par cette première heure de classe. Sa façon d'être, d'agir, de percevoir, 
d'éprouver, de connaître suite à cette expérience pouvait être caractérisée par une position d'attente et de résignation. A l'issue de cet épisode, elle pense tester de nouvelles pistes d'action en s'inspirant des observations vidéo et des analyses proposées par le formateur.

Du point de vue des processus identitaires les extraits relatifs à cette modalité «sortie d'impasse» montre que certaines "doxas» partagées par beaucoup de débutants peuvent impacter la trajectoire de formation des débutants et de fait impacter les processus de construction identitaire.

\section{Transitions de phases: Jeanne, professeur stagiaire, enseignement primaire}

Cet extrait concerne Jeanne, professeur titulaire première année dans l'enseignement primaire: «Le CPC [Conseiller Pédagogique de Circonscription] est passé [hier pour la première fois], il m'a dit au niveau de l'autorité, ils ne bougent pas... J'ai soufflé [...] Depuis j'ai pu penser et voir autre chose que leur agitation et leurs bavardages " [Jeanne, enseignement primaire, octobre]. L'interaction ici avec un $\mathrm{CPC}$ semble témoigner de cette focalisation en amont de la visite. La débutante affirmait en amont de cette visite de formation être focalisé sur l'agitation des élèves, elle apparaissait très sensible à ces aspects. Elle indiquait également attendre de cette interaction un retour sur ce volet particulier estimant ne pas être totalement objective sur ce que doit être un climat de classe acceptable. L'appréciation du CPC «ils [les élèves] ne bougent pas» est pour elle un épisode clef à double titre. Rassurée sur son activité avant la visite, elle semble d'une part libérée d'un poids. D'autre part, ce moment semble ouvrir de nouvelles perspectives sur les aspects liés à la gestion des apprentissages. Jusque-là réticente à orienter son activité en ce sens, elle est, suite à cette visite, plus disposée à observer d'autres indices davantage liés à l'apprentissage qu'à la gestion de classe. L'activité en amont et en aval de ces expériences se caractérise par une certaine continuité dans les observations en classe que nous avons pu faire, cependant la focalisation relative à la gestion de classe de Jeanne est plus ponctuelle dans le décours de son activité à la suite de cette visite.

Si cette focalisation a été souvent décrite comme première chez les enseignants débutants, il nous semble ici important de souligner l'attente d'une validation de ces aspects comme préalable à une orientation de l'activité sur la gestion des apprentissages qui se traduit dans les faits par une attention accrue portée aux productions des élèves et aux procédures qu'ils semblent mobiliser pour réaliser les tâches scolaires.

Reconstructions d'édifices: Raphaël, professeur stagiaire d'Histoire-Géographie L'extrait d'entretien concerne Raphaël, professeur stagiaire d'Histoire-Géographie. Il est relatif à un entretien de conseil réalisé par un formateur IUFM à la suite de l'observation d'une leçon sept mois après la rentrée des classes. Cette visite du formateur a été commentée comme un épisode de forte déstabilisation pour le jeune professeur. 
En amont de cette visite, nos observations et nos entretiens ont fait apparaître que Raphäl a rapidement trouvé ses marques face aux élèves. Son début d'année est vécu de façon positive avec le sentiment d'avoir trouver des repères et d'avoir construit des habitudes de fonctionnement et d'interaction avec les élèves. Quelques jours avant la visite, il considère que si les premières semaines ont été marquées par une recherche de repères sur ce que les élèves sont susceptibles de comprendre et de réaliser; les semaines et mois suivants ont consisté à installer un climat de travail collaboratif. Ce dernier encourageait une écoute mutuelle et suscitait les interactions entre les élèves. A la suite de la visite Raphaël semble fortement déstabilisé: "J'avais l'impression d'avoir installé pas mal d'habitudes, de choses avec mes élèves, d'avoir installé des quilles et là mon formateur [Formateur IUFM] j'ai l'impression qu'il vient de jeter une boule de bowling en plein milieu de mes quilles" [Raphaël, Histoire-Géographie, mars]. Cet épisode marque le passage soudain d'une impression de stabilité à une perte totale de repères: «Là j'ai eu l'impression qu'il me jetait une boule de bowling en plein milieu de mes quilles [...]. Il a réussi à me faire réagir et en une semaine et demiej jai beaucoup remué». Les remarques faites à cette occasion l'ont affecté dans la mesure où il était jusque-là persuadé d'avoir réussi son début d'année. Il avait stabilisé une façon d'interagir avec les élèves qu'il estime devoir grandement remettre en cause au moment même de ce temps de formation. Cet évènement a amené Raphaël à d'importantes transformations de son activité en classe. La détresse du débutant, estimée grande, semble liée aux enjeux identitaires associés à ces habitudes d'interactions édifiées durant les premières semaines d'enseignement. Soucieux de rectifier au plus vite ce qu'il estimait être des erreurs, Raphaël a tenté d'appliquer à la lettre les conseils prodigués dès le lendemain de la visite. Seulement, les changements opérés ont fait que les élèves étaient perdus, ne retrouvant pas les habitudes de travail établies jusque-là. Cette tentative d'application des conseils prodigués du jour au lendemain s'est soldée par un retour quasi immédiat aux pratiques antérieures. Ce n'est que par la suite que des transformations ponctuelles ont été travaillées par retouches successives.

Les extraits relatifs à cette modalité "reconstruction d'édifices» montre que la remise en cause soudaine et massive des façons d'être, d'agir, de percevoir, d'éprouver et de connaître suite à cette visite suppose également une évolution de la façon d'enseigner qui elle ne peut pas être soudaine et brutale. La description de tels cas souligne à nos yeux les synergies asynchrones entre la transformation des gestes professionnels (introduire une problématique en questionnant les élèves) et la nécessité de rester la même personne aux yeux des élèves (nous avons pu observer un élève affirmant, lors de la leçon suivant la visite du formateur, être perdu et ne plus reconnaittre son professeur). Les transformations des habitudes d'interactions apparaissent donc plus lentes que les transformations des gestes professionnels. Asynchrones, ces évolutions semblent d'autant plus sous la dépendance des habitudes d'interactions que ces habitudes sont établies et fonctionnelles pour l'entité "classe», c'est-à-dire aussi bien pour 
le professeur que pour les élèves. Les difficultés sont dès lors multiples pour les enseignants débutants sur le plan des processus identitaires. Il est difficile pour eux de percevoir après coup les limites des pratiques qu'ils avaient stabilisé parfois depuis de nombreuses semaines. A la culpabilité qu'ils ressentent dans ces circonstances s'ajoute la nécessité de recomposer leur façon d'enseigner tout en la conservant pour partie. En effet, lorsqu'ils transforment leur façon d'enseigner, il s'agit pour eux de rester dans une certaine mesure le professeur que les élèves reconnaissent tout en devenant progressivement un autre professeur.

\section{Discussion}

Cette analyse des transformations de l'engagement apparait comme un élément susceptible de rendre compte des imbrications entre processus identitaires et processus de formation. Elle montre la complexité et la fragilité des processus de construction identitaire qui reste très difficilement appréhendable par les formateurs.

L'enjeu est ici de savoir si ces transformations de l'engagement des enseignants débutants, imprévisibles, sont pour partie modélisables. Étudier la nature de ces expériences est à nos yeux une voie prometteuse susceptible d'aider les formateurs à accompagner les débutants face aux aléas des situations de début dans le métier.

\section{Jouer sur la réversibilité des situations}

Les données font apparaître des enjeux forts autour de la façon dont les débutants s'emparent de ce que nous qualifions de "doxas" professionnelles, principes généraux véhiculées dans et hors la formation. Ces «doxas» présentent des séquences d'action et qualifient dans le même temps les rapports de causalité entre elles. Si l'on se réfere à l'affirmation suivante "Rater sa première heure de cours c'est rater son année», on observe qu'une première séquence d'action (courte) «rater sa première heure de cours» est mis en relation avec une autre séquence d'action (plus longue) «rater son année». L'irréversibilité affirmée de cet enchainement de séquences peut configurer durablement le processus de formation des débutants. Si les enseignants expérimentés semblent composer avec ces «doxas», il n'en est pas autant des débutants. Pouvoir remettre en jeu ou modérer la portée de séquences d'action perçues par ces derniers comme irréversibles «rater sa première heure de cours c'est rater son année» ou comme incontournables: «le silence est un préalable au travail» revêt à nos yeux une importance dans la mesure où ces dernières tendent à figer l'activité des débutants et son développement.

Le repérage de ces doxas nous semble une piste de travail possible. Les formés avec lesquels nous avons travaillé sont capables d'en verbaliser certaines comme "pas un sourire avant Noël», «ne jamais tourner le dos aux élèves». Elles peuvent 
être générales ou plus spécifiques aux disciplines enseignées et au degré d'enseignement concerné. D'autres encore sont propres à des contextes d'enseignement où aux caractéristiques des élèves.

\section{Accélérer la survenue de processus jugés favorables}

Certains processus porteurs d'enjeux identitaires forts nous semblent à reconsidérer en vue d'être optimisés. Placés le plus souvent dans des situations duelles de formation ou d'accompagnement, les débutants découvrent lentement les différents styles d'enseignement. Ils construisent leur identité professionnelle par adhésion ou prise de distance vis-à-vis des enseignants et formateurs qu'ils rencontrent séparément, les uns après les autres. Ces confrontations, plus souvent successives que simultanées, supposent à la fois une «mise en mémoire des styles " et un temps important de découverte de la "palette des styles» (Serres, Ria, Adé \& Sève, 2006). Soucieux de trouver leur propre style, les débutants profitent grandement de tous les moments de formation susceptibles de mettre en perspective des façons d'enseigner diverses. Il semble donc pertinent de réunir en formation de manière plus systématique les conditions d'émergence de ces processus jugés favorables pour le développement de l'identité professionnelle des enseignants débutants.

Penser la progressivité des questions professionnelles Nous jugeons utile de distinguer les étapes entre stabilisation d'une pratique d'enseignant débutant et stabilisation d'une pratique d'enseignant expérimenté. La formation ne distingue pas ou pas assez à nos yeux ce que l'on peut nommer des questions de métier et des questions de débutants ${ }^{1}$. Nous soulignons ce point dans la mesure où ceci nous semble avoir une portée conséquente sur les processus identitaires. Sans cette distinction, les débutants entrent dans le métier en visant d'emblée des questions de métier souvent hors de leur portée. Ceci peut être exemplifié en s'appuyant sur les situations de conseil. Les formés semblent bien souvent en difficulté d'établir des priorités face à la multitude des conseils qu'ils reçoivent en cascade (Serres \& Ria, 2007). Si la plupart de ces conseils apparaissent spécifiques des questions que les débutants sont en mesure de se poser, d'autres semblent renvoyer bien plus largement à des questions de métier en débat dans la communauté professionnelle. Cette distinction nous semble à même d'opérer une remise en ordre des questions professionnelles cruciales pour des débutants, ou du moins d'attirer l'attention des formateurs sur les risques liés au fait de susciter trop précocement chez les débutants des questions que les plus expérimentés peinent collectivement à se poser et à travailler.

\section{Tenir compte des dynamiques simultanément à l'œuvre} Étudier l'activité en essayant de décrire des dynamiques simultanément à l'œuvre amène plusieurs réflexions sur leurs caractéristiques propres et leurs interdépendances. L’engagement semble évoluer rapidement et fluctuer grandement sous 
l'influence du sentiment d'identification à la communauté enseignante, sous l'influence des jugements qu'ils portent sur leurs premières expériences et sous l'influence des "doxas», de principes généraux, véhiculées dans et hors de la formation. Ce qui semble sur ce point important de repérer est d'une part, la sensibilité des évolutions de l'engagement et d'autre part la portée de ces évolutions qui reconfigurent parfois de façon conséquente et durable l'engagement des débutants dans leur formation.

Si l'apprentissage du métier passe par des périodes d'immersion, nous constatons que cette modalité de formation oriente grandement le processus de formation. L'attention que les débutants portent par exemple à la question de l'autorité, point clef de reconnaissance identitaire dans la communauté enseignante (Dubet \& Martucelli, 1996), fait qu'ils tendent à redéfinir le métier en limitant ce dernier à une activité de contrôle d'un groupe par un individu. La portée de ces expériences de contrôle du groupe sur les processus identitaires est grande. En ce sens, l'alternance et les relations de dépendance entre activité constructive et productive (Rabardel \& Samurcay, 2004) semblent constituer un enjeu clef insuffisamment conceptualisé et trop souvent laissé sous la dépendance d'apprentissages opportunistes se développant dans des interstices de l'activité (Olry, 2002). En transformant le réel, le professeur débutant se transforme lui-même. Et ces deux sortes d'activités, productive et constructive, constituent un couple inséparable. Dans les situations de travail (prises dans des dispositifs de formation qui tentent d'orienter ces situations ou de les aménager) le but de l'action reste bien l'activité productive; et l'activité constructive n'est qu'un effet, qui n'est généralement ni voulu ni conscient (Pastré, 2006). Si activité et apprentissage sont indissociables, il nous semble important que les dispositifs de formation intègrent une réflexion sur les itérations entre ces deux sortes d'activés de façon à ce que l'activité constructive soit le plus largement possible accompagnée.

\section{Conclusion}

Nous souhaitons en conclusion insister sur le fait qu'il ne suffit pas de décrire quelques cas de reconfigurations de l'engagement des enseignants en formation pour rendre compte des processus identitaires en jeu. Dans les données récoltées, les enseignants débutants font l'expérience d'une nouvelle cohérence, ils affirment avoir changé ou pouvoir agir différemment en raison d'une perspective renouvelée sur leur environnement. Cette expérience de reconfiguration de leur engagement est constituée en entité. Elle est distinguée, discrétisée dans le flux de leur expérience (Perrin, 2011) et constitue dès lors une perturbation susceptible de modifier de manière importante leur activité. Lors de ces bascules, une reconfiguration de ce qui faisait l'unité de leur expérience s'opère. C'est à ce titre que nous pensons que la description de ces dynamiques semble être un support 
intéressant pour appréhender les processus identitaires en formation.

Par ailleurs, à la suite de cette étude, il nous semble possible de repérer dans les parcours des débutants des expériences similaires, des difficultés partagées, des passages obligés, des engagements dans des impasses, des évolutions ou transitions fréquemment observées. Ce travail nous semble à systématiser à partir d'analyses plus conséquentes dans la lignée des travaux décrivant comment les débutants en formation et lors de leurs débuts dans le métier recherchent des ajustements momentanément acceptables (Perez-Roux, 2006), comment ils cheminent pas-à-pas en stabilisant des états transitoires (Ria, 2010). Sans tomber dans une perspective déterministe mais sans tomber à l'inverse dans une perspective dans laquelle tout serait contingent et imprévisible, nous pensons que ce repérage devrait permettre de cerner des phénomènes en vue de les considérer comme de possibles passages critiques ou à l'inverse comme de possibles leviers pour favoriser le processus de formation des débutants et leurs débuts dans le métier.

\section{Notes}

1 Distinction issue du travail mené sur la plateforme Néopass@ction sur la thématique consignes et rituels à la maternelle en collaboration avec Roland Goigoux et Patrick Picard.

\section{Bibliographie}

Baillauquès, S. (2002). Identité et responsabilité. Ou comment la responsabilité vient aux enseignants débutants. Recherche et formation, 41, 65-82.

Boutet, M. \& Pharand, J. (2008). L'accompagnement concerté des stagiaires en enseignement. Québec: Presses universitaires du Québec.

Chaliès, S. Bertone, S. Flavier, E., Durand, M. (2008). Effects to collaborating mentoring on the articulation of training and classroom situations: a case study in the french school system. Teaching and Teacher Education, 24(3), 550-563.

Barbier, J. M., Bourgeois, E., De Villiers, G. et Kaddouri, M. (2006). Constructions identitaires et mobilisation des sujets en formation. Paris: L'Harmattan

Dubet, F. \& Martucelli, D. (1996). A l'école. Sociologie de l'expérience scolaire. Paris: Seuil.

Durand, M. (2008). Un programme de recherche technologique en formation des adultes. Une approche enactive de l'activité humaine et l'accompagnement de son apprentissage développement. Education et Didactique, Vol. 2(2), 69-93.

Durand, M. (2009). La conception d'environnement de formation sous le postulat de l'enaction. In M. Durand, \& L. Filliettaz (Éd.), Travail et formation des adultes (pp. 191-225). Paris: PUF.

Goigoux, R., Ria, L. \& Toczek-Capelle, M.-C. (2009). (Éd.). Les parcours de formation des enseignants débutants. Presses Universitaires Blaise Pascal.

Gouillaud, V. (2001). Les attentes et les espérances de formation chez les futurs enseignants. In Baillauquès, S. Dupuis, P.-A., Ferry, G., Kempf, M. et Tournier, F. (Éd.), La personnalisation d'une formaton professionnelle. Le cas des professeurs des écoles. Paris: INFP-IUFM.

Malet, R. (2008). La formation des enseignants comparée. Identité, apprentissage et exercice professionnels en France et en Angleterre. Berne: Peter Lang.

Malet, R. (1998). L'identité en formation. Phénoménologie du devenir enseignant. Paris, L'Harmattan.

Olry, P. (2002). Tempo de l'activité et apprentissages opportunistes au travail. Revue française de Pédagogie, 138, 19-28. 
Pastré, P. (2006). Apprendre à faire. In E. Bourgeois \& G. Chapelle (Éd.), Apprendre et faire apprendre (pp. 109-121). Paris: PUF.

Perez-Roux, T. (2006). Construction des compétences professionnelles chez les enseignants débutants: entre logique de formation, logique des acteurs et effets de contexte», colloque CNAM: usages sociaux de la notion de compétences. Paris.

Périsset, D. (2006). La formation sur le terrain à la HEP-VS: le rôle des praticiens-formateurs. Enjeux pédagogiques, bulletin de la Haute école pédagogique de Berne, du Jura et de Neuchâtel, 3, 19.

Perrin, N. (2011). Une approche enactive de la construction de connaissances en formation professionnelle initiale des enseignants. Analyse du "cours de languaging" au sein d'un dispositif de simulation-analyse. Thèse en sciences de l'éducation, Université de Genève, Genève.

Samurçay R. \& Rabardel P. (2004). «Modèles pour l'analyse de l'activité et des compétences, propositions». In R. Samurçay \& P. Pastré (dir.), Recherches en didactique professionnelle (pp. 163-180). Toulouse, Octarès.

Rayou, P. \& Ria, L. (2009). Former les nouveaux enseignants. Autour des statuts, de l'organisation et des savoirs professionnels. Education et Sociétés, 23, 79-90.

Ria, L. (2010). Des pistes prometteuses pour la conception de dispositifs de formation des enseignants débutants. In R. Goigoux, L. Ria \& M.C. Toczek-Capelle (Éd.), Les parcours de formation des enseignants débutants, (pp. 325-333). Clermont-Ferrand: Presses Universitaires Blaise-Pascal.

Ria, L., Serres, G. \& Leblanc, S. (2010). De l'observation vidéo à l'observation in situ du travail enseignant en milieu difficile: étude des effets sur des professeurs stagiaires. Revue Suisse des Sciences de l'Éducation. 32(1)/2010, 105-120.

Ria, L., \& Leblanc, S. (2011). Conception de la plateforme de formation Néopass@ction à partir d'un observatoire de l'activité des enseignants débutants: enjeux et processus. Activités, 8(2), pp. 150-172, http://www.activites.org/v8n2/v8n2.pdf

Rouve, M-E. \& Ria, L. (2008). «Analyse de l'activité professionnelle d'enseignants néo-titulaires en réseau "ambition réussite»: études de cas», Travail et formation en éducation [En ligne], 1 | 2008, mis en ligne le 15 décembre 2008, Consulté le 14 décembre 2011. URL: http://tfe.revues.org/index $565 . \mathrm{html}$

Saujat, F. (2004). Comment les enseignants débutants entrent dans le métier. Formation et pratiques d'enseignement en questions. Revue des HEP de Suisse Romande et du Tessin, 1, 97-106.

Serres, G. (2006). Analyse de la construction de l'expérience professionnelle au gré des diverses situations de formation initiale des enseignants du second degré. Thèse en sciences de l'éducation, Université de Clermont-Ferrand 2, France.

Serres, G., Ria, L., Adé, D., \& Sève, C. (2006). Apprend-on vraiment à intervenir en formation initiale? Prémisses du développement de l'activité professionnelle dans les dispositifs de formation en alternance. STAPS, 72(2), 9-20.

Serres, G. \& Ria, L. (2007). Questionner la formation des enseignants à partir de la description des trajectoires des formés. Revue des Hautes Écoles pédagogiques et Institutions assimilées de Suisse Romande et du Tessin, Vol. 6, 99-119.

Theureau, J. (2004). Le cours d'action: méthode élémentaire. Toulouse: Octarès.

Theureau, J. (2006). Le cours d'action: méthode développée. Toulouse: Octarès.

Theureau, J. (2009). Le cours d'action: méthode réfléchie. Toulouse: Octarès.

Mots-clés: Formation, reconfigurations, engagement, processus identitaires 


\section{Dynamiken des Engagements von Junglehrpersonen in der Ausbildung und die Erschliessung von Identitätsprozessen}

\section{Zusammenfassung}

Mit Bezug auf die Fragestellungen dieses Themenheftes schlägt dieser Beitrag vor, Bildungserfahrungen von Junglehrpersonen zu betrachten, bei denen im Verlauf ihrer Berufseinstiegsphase Veränderungen ihres Engagements identifiziert werden können. Das gewählte Vorgehen legt besonderen Wert auf die dynamische Dimension der betrachteten Phänomene, weshalb diese durch Längsschnittstudien beschrieben werden. Drei Arten der Transformation resp. der Rekonfiguration des Engagements von Junglehrpersonen werden charakterisiert: «Auswege aus (den) Sackgassen», «Übergänge» und «umfangreiche Rekonstruktion». Ein besseres Verständnis der Identitätsprozesse, die sich während dieser Rekonfigurationen ergeben, scheint vielversprechende Perspektiven für die Ausbildung zu eröffnen.

Schlagworte: Lehrerbildung, Berufseinstiegsphase, Rekonfiguration, berufliche Identitätsprozesse

\section{Dinamiche di implicazione nella formazione degli insegnanti neofiti e studio dei processi identitari}

Riassunto

In risposta agli stimoli di questo numero tematico, questo articolo si propone di presentare l'analisi di esperienze formative di insegnanti alle prime armi, durante le quali sono reperibili delle traformazioni nelle loro modalità di implicazione. L'approccio scelto accorda un'importanza particolare alla dimensione dinamica dei fenomeni descritti, privilegiando dunque degli studi di tipo longitudinale. Tre modalità di trasformazione e riconfigurazione dell'implicazione degli insegnanti neofiti sono state caratterizzate: "uscita da un'impasse», "transizione» o «ricostruzione massiva». Una migliore comprensione dei processi identitari in gioco durante tali riconfigurazioni ci sembra offrire piste promettenti per la formazione.

Parole chiave: Processi identitari, insegnanti neofiti, dinamiche di implicazione, attività 


\section{Commitment dynamics of novice teachers in training and understanding of identity processes}

\section{Summary}

In reference to the challenges of this special issue, the paper gives an account for learning experiences of beginning teachers and of the changes in their commitment identifiable in the process. The approach based on longitudinal studies places particular importance on the dynamic dimension of phenomena over the training period. Three modes of changing and reconfigurating the novice teachers' commitment were identified: "finding a way out», "transitising" and «massively reconstructing». A better understanding of identity processes involved in these reconfigurations seems likely to open promising avenues for training.

Kewords: Identity processes, preservice teachers, commitment dynamics, activity 